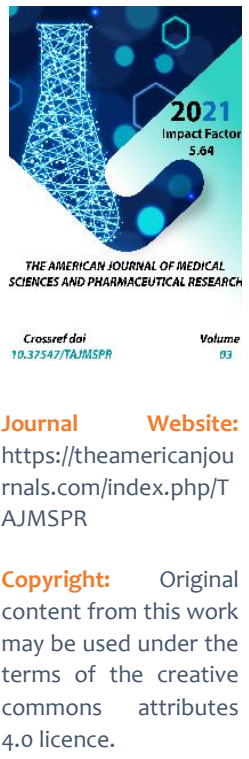

\title{
Change Of Morphological Structure Of The Mucosa In Polypragmasia
}

\author{
Toshmamatov Bakhtiyor Norbekovich \\ Assistant, The Department Of Normal And Clinical Anatomy Of The Samarkand State Medical \\ Institute, Uzbekistan \\ Teshaev Shukhrat Zhumaevich \\ Scientific Adviser - Doctor Of Medical Sciences, Professor, The Department Of Clinical Anatomy \\ (OSTA) And Forensic Medical Expertise, Uzbekistan
}

\section{ABSTRACT}

In the contemporary world, there is a rapid increase in the invention and application of a large number of medications (drugs) in practical health care, which, on the one hand, can cure and/or enhance the patient's condition, but also do substantial harm to his health. The purpose of this study was to add to the body of knowledge on polypragmas that develop following nonsteroidal therapy in rats. Gastric tissue was morphologically examined in 55 rats to attain the aim. The findings revealed that nonsteroidal therapy resulted in an increase in the amount of mucous membranes and lymphoid follicles, proliferation of vascular wall cells, and poor stratification of connective tissue cells in the stomach.

\section{KEYWORDS}

Stomach, non-steroidal anti-inflammatory drugs (NSAIDs), morphology, and experiment.

\section{INTRODUCTION}

The study of various aspects of pathogenesis and morphogenesis of gastrointestinal tract disease constitutes a significant part that gradually grows into a separate, essential field of medical science. To date, various changes in the stomach mucous membrane are described, arising from the effects of various types of damaging factors: chemical, physical, infectious; At the same time, morphological changes of the structure of the mucous membrane of the stomach of the GT, with the effects of various types of damaging agents, have their peculiarities [Minushkin O.N. et al., 2013; Karateev A.E., 2011]. Morphological changes of SGD and pathogenesis of chronic gastritis of the disease have their peculiarities 
for different forms of gastritis [Minushkin O.N. et al., 2013]. It is generally accepted that Helicobacter pylori, associated gastritis, is the most common, but the so-called NSAID gastropathy (one of the varieties of chemical gastritis, type $\mathrm{C}$ ), damage to the gastric mucosa caused by the intake of non-steroidal anti-inflammatory drugs (NSAIDs), is no less relevant. Non-steroidal anti-inflammatory drugs (NSAIDs) are among the most commonly prescribed drugs by physicians to treat various conditions in patients of all age groups [Hawkey C.I. et al., 2011]. Various clinical symptoms of inflammatory diseases combined with limited possibilities of etiotropic therapy, a large number of symptomatic agents became the causes of polyprogmasia. Unreasonable treatment often slows down the natural healing process. Recently, the gastrointestinal tract is considered an essential component of the general defense of the body. [Nasyrova S.Z. et al., 2021].

Despite the study, questions related to the features of changes in the proliferation of the epithelium of the gastric mucosa with prolonged use of NSAIDs remain poorly studied and controversial.

\section{PURPOSE OF THE STUDY}

To study the morphometric parameters of the stomach of healthy white rats in postnatal ontogenesis from 3 months of age to 6 months and the simultaneous use of several antiinflammatory drugs.

\section{MATERIALS AND METHODS}

The work was carried out on white outbred male rats aged from 3 to 6 months in the usual conditions of the vivarium. The timing of the study was chosen by us based on data on the structural and functional characteristics of the stomach and age periodization of laboratory animals. The morphological changes of the gastric mucosa were studied experimentally in 40 white outbred male rats weighing 160-220g. Autopsy materials were used as material for the study. For general morphology, pieces were excised from each stomach and solidified in $10 \%$ neutral formalin. After washing for $2-4 \mathrm{~h}$ in running water, it was dehydrated in concentrated alcohol and chloroform, then embedded in paraffin and prepared blocks. On paraffin blocks, sections of 5-7 $\mu \mathrm{m}$ were cut, stained with hematoxylin and eosin. Semi-thin $1 \mu \mathrm{m}$ sections were obtained from Epon bricks on a Leyka ultramicrotomy. Histological preparations were examined under 10, 20, 40 lenses of a light microscope and the necessary areas were photographed. In a rat under ether anesthesia, the abdominal cavity was decapitated, and morphological changes in the stomach were studied. After macro anatomy, the stomach was isolated for further study. The experiments on laboratory animals were carried out following the Declaration of the International Medical Association, adopted in Helsinki in 1964 and completed in 2013.

The isolated stomach was fixed in solution and poured into paraffin. Then, incisions with a 6-7 $\mu \mathrm{m}$ were prepared and stained using the hematoxylin-eosin and Van Gieson methods. Histological preparations were examined under 10, 20, 40 lenses of a light microscope, and the necessary areas were photographed.

\section{RESULTS AND DISCUSSION}

Our investigation was about the morphological and morphometric changes in rats' stomach, characterize its various departments, and 
determine the changes in cutting fluids. We studied a gradual change in the structure of morphometric parameters of parts and layers of the stomach wall of white rats from 3 months to 6 months of age with the simultaneous use of up to 5 different types of anti-inflammatory drugs. Normally, a morphometric study of the wall of the gastric mucosa showed that the number of lymphoid follicles changes depending on age, which averaged $32.4+4.7$, in 3 months $37.4+5.7,4-6$ months $76.4+7.6$. This increase in the number of cells of lymphoid follicles continues in the following months. With long-term use of NSAIDs in rats, the following changes were observed: lymphoid follicles of the stomach were undeformed, hyperplastic atypically and unevenly. Their boundaries are not determined; lymphocytes are located around the postcapillary venules. As a result of studies in the stomach wall of rats receiving antiinflammatory drugs, changes of a destructive nature were found, depending on the number of drugs. Their boundaries are not determined; lymphocytes are located around the postcapillary venules. As a result of studies in the stomach wall of rats receiving antiinflammatory drugs, changes of a destructive nature were found, depending on the number of drugs. Destructive changes are characterized by edema of the stroma of the villi, their desquamation into the lumen of the stomach, a decrease in the height of the villi, which were observed in the second group when using several types of anti-inflammatory drugs. At the same time, the observed increase in the number of goblet cells in the villi. Presumably, this is due to an increase in degenerative processes in epithelial cells under the influence of polyphragmasy.

Microscopic examination of the antrum of the stomach of white rats of the control group showed that the mucous, muscular, and serous membranes are well developed and have distinct boundaries. The mucous membrane contains many large diameter glands and smaller crypts. On the surface of the villi and crypts are cylindrical epithelial cells with oval nuclei. Among the epithelial cells are goblet cells, the most significant number of which is observed in crypts.

According to our results, the middle part of the stomach wall study shows a different degree of morphological changes when exposed to different amounts of drugs. It was found that after exposure to drugs, the size and ratio of structural components in the walls of the stomach noticeably change. In the gastric mucosa of the experimental group of animals, intense desquamation of epithelial cells into the stomach lumen was observed at the tops of the villi. The stroma of the villi is edematous, it contains a large number of macrophages and lymphocytes, and necrosis of the villi is observed in some areas (Fig. 1). 


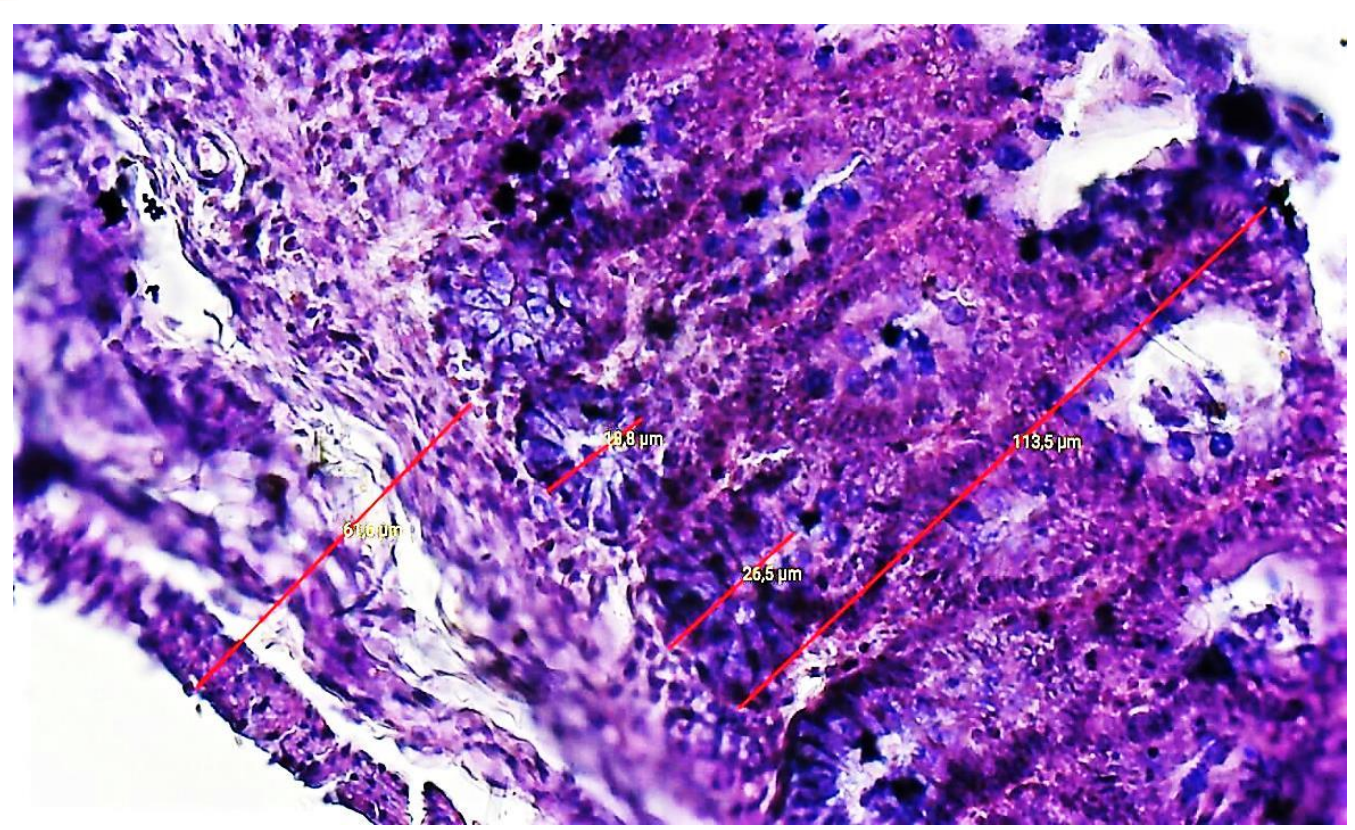

Figure № 1. In the stomach mucous membrane, occurs edema, and the interstitial tissue is thickened due to the proliferation of fibrous connective tissue. Stain: Hematoxylin and eosin. 10x10.

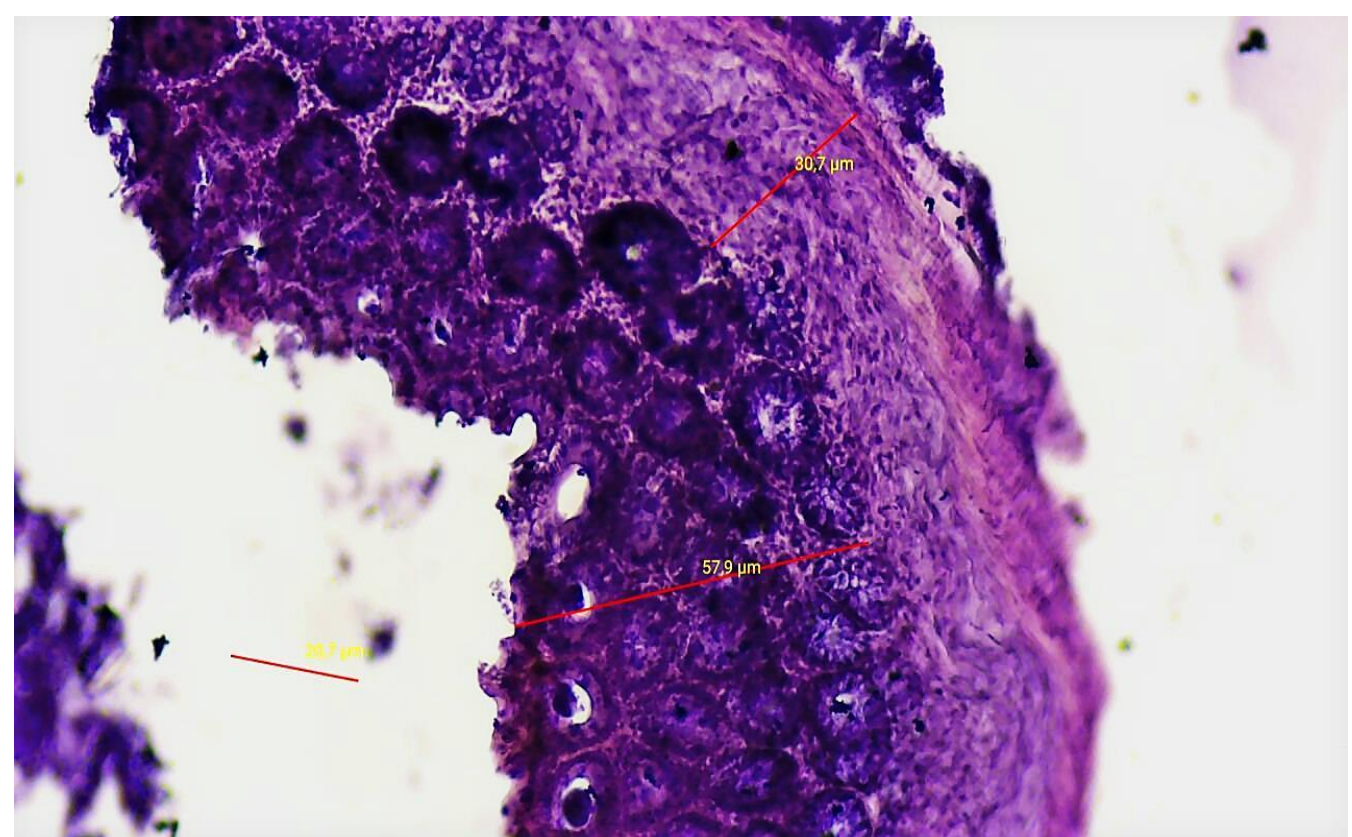

Figure № 2. Dystrophic and necrobiotic changes in epitheliocytes with polyphragmasy, lymphohistiocytic infiltration. Stain: Hematoxylin and eosin. 10x10. 


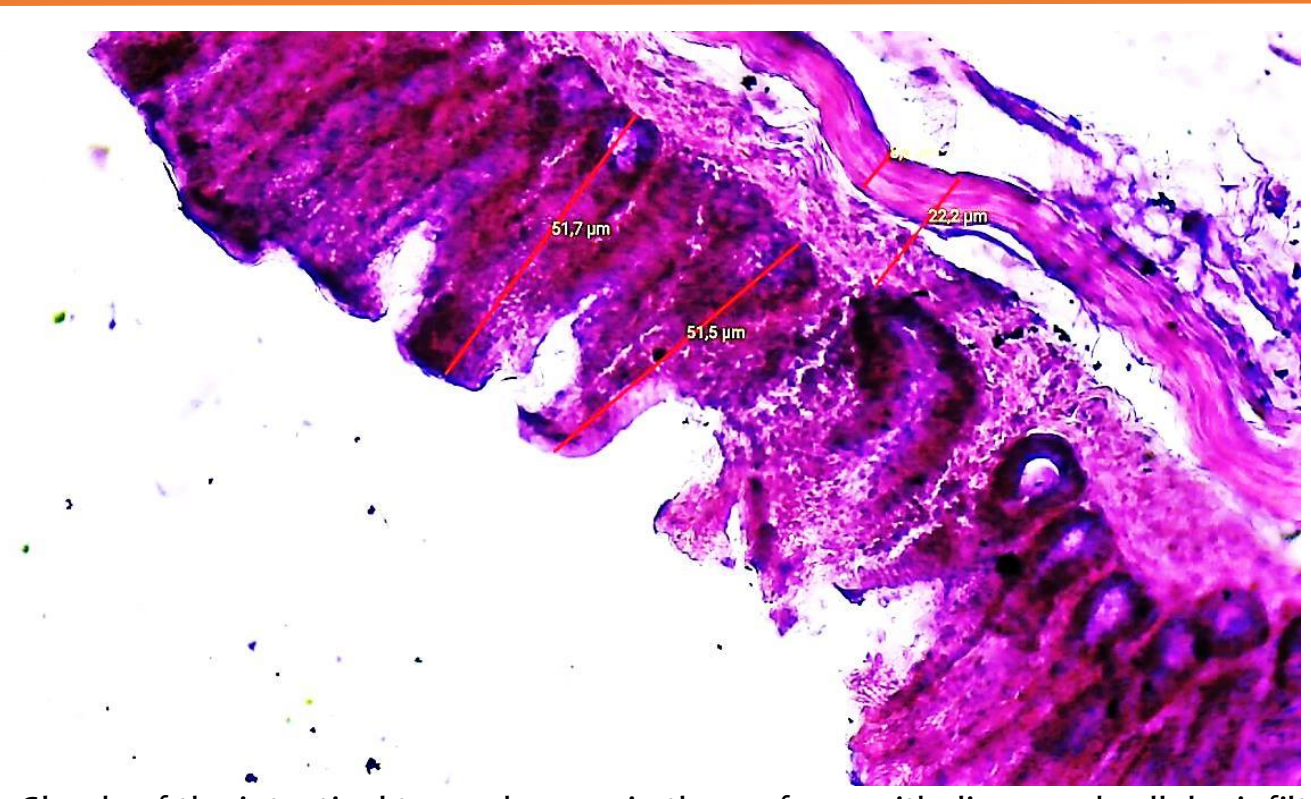

Figure № 3. Glands of the intestinal type, changes in the surface epithelium, and cellular infiltration of lymphocytes in the gastric mucosa. Stain: hematoxylin and eosin, $10 \times 40$.

When taking NSAIDs, regeneration also developed atrophy of the mucous membrane, even more intensively, but the formation of normal glandular elements was blocked, and instead of them, various poorly differentiated glandular cells appeared.
Moreover, both atrophic and dystrophic changes in the mucosa deepened and prolonged use of NSAIDs in rats pathomorphologically, against the background of which catarrhal-desquamative, necroticulcerative gastritis developed. 


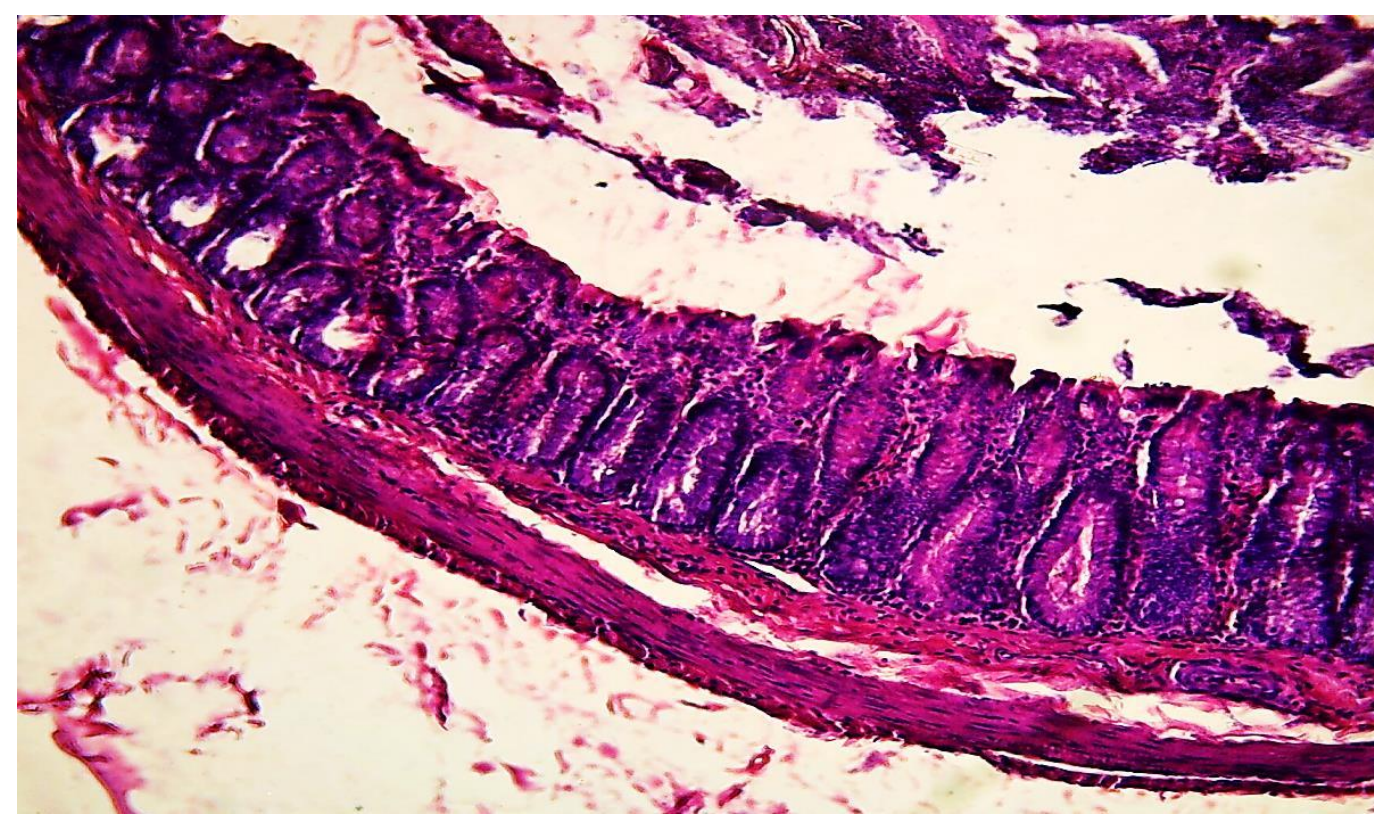

Figure № 4. When taking a non-steroidal preparation, the destruction of epithelial cells manifests in the stomach and a decrease in the secretory activity of mucous membrane cells, staining with hematoxylin and eosin, $10 x 10$.

\section{CONCLUSION}

The above facts insist on the need for further in-depth study of factors that adversely affect the mucous membrane of the stomach in various pathologies of the gastrointestinal tract. With the long-term application of more than 5 NPAs in rats pathomorphologically, catarrhal-desquamative, necrotic-ulcerative gastritis developed.

\section{REFERENCES}

1. Agapova N.G. (2007). About medicinal gastroduodenal ulcers. Mystetstvo lykuvannya. (2)38.

2. Gelfand B.R., Guryanov A.V., Martynov A.N. et al. (2005). Prevention of stress damage to the gastrointestinal tract in critically ill patients. Consilium medicum. 7(6). pp. 464-67.
3. Zhuraeva G.B. (2015). The activity of pathomorphological changes in the intestinal lymphoid tissue under the influence of acute intestinal infections. Yakutsk Medical Journal. 2(50) pp. 6367.

4. Zhuraeva, G.B., Saidov, A.A., \& Israilov, R.I. (2015). The dependence of morphological and morphometric changes in the mucous membrane in acute intestinal infection on the type of pathogen. Youth Innovative Bulletin, 4(1), pp.193-195.

5. Karateev A.E. (2006). Treatment and drug prevention of NSAID gastropathy: basic provisions. Pharmateka, 6(121). pp.37-45.

6. Sapin M.R. (2012). The lymphatic system and its role in immune processes. Morphology, 141(3), p. 139.

7. Safonova M.A. (2009). Hygienic assessment of the impact of 
technogenic environmental factors on the development of chronic gastroduodenitis in children (on the example of the Perm Territory): Avtoref.dis.kan.nauk Perm,; 24.

8. Teshaev Sh.Zh., Khudoiberdiev D.K., Teshaeva D.Sh. (2018). The impact of exogenous and endogenous factors on the stomach wall. Problems of biology and medicine. 4(104). pp. 212-214.

9. Teshaev Sh.Zh., Toshmamatov B.N. (2021). Morphofunctional characteristics of the stomach in polypharmacy. Journal of a new day in medicine. 3. pp.227-232.

10. Chava S.V. (2004). Reactive changes in immune structures in the wall of the small intestine. Morphological statements, 1-2, p. 114-115.

11. Ahrorova, K. D. (2021). Morphofunctional properties of the lymphoid structures of the spleen in norm and under the influence of various factors. Academicia: An International Multidisciplinary Research Journal, 11(1), 459-465.

12. Andersson $\mathrm{T}$, Andren $\mathrm{N}$, Cederberg $\mathrm{C}$, et al. (1990). Bioavailability of omeprazole as enteric coated granules in conjunction with food on the first and seventh day of treatment. Drug Invest. 2. pp.184-88.

13. Andersson T, Cederberg C, Heggelund A. (1991). The pharmacokinetics of single and repeated once daily dose of 10, 20 and $40 \mathrm{mg}$ omeprazole as enteric coated granules. Drug Invest; 3, pp.4552.

14. Siurala M., Voris K. Gastritis. (1999). In: Scientific Foundations of Gastroenterology, edited by W.Sircus and A. N. Smith, 357-369, London, William Heinemann Medical Books Ltd. 